\title{
A layered approach to neck lift
}

\author{
Andrew L. Weinstein ${ }^{1}$, Foad Nahai ${ }^{2}$ \\ 'Division of Plastic Surgery, Emory University School of Medicine, Atlanta, GA 30327, USA. \\ ${ }^{2}$ The Center for Plastic Surgery at MetroDerm, Atlanta, GA 30342, USA.
}

Correspondence to: Dr. Foad Nahai, The Center for Plastic Surgery at MetroDerm, 875 Johnson Ferry Rd NE, Suite 150, Atlanta, GA 30342, USA. E-mail: fnaha02@emory.edu

How to cite this article: Weinstein AL, Nahai F. A layered approach to neck lift. Plast Aesthet Res 2021;8:11.

http://dx.doi.org/10.20517/2347-9264.2020.192.

Received: 14 Oct 2020 First Decision: 4 Dec 2020 Revised: 8 Dec 2020 Accepted: 19 Jan 2021 Published: 7 Feb 2021

Academic Editor: Oscar M. Ramirez Copy Editor: Yue-Yue Zhang Production Editor: Xi-Jun Chen

\begin{abstract}
Neck aesthetics are a vital and indispensable component of cervicofacial beauty. Cosmetic deformities may be due to congenital or acquired etiologies and successful management depends on accurately diagnosing the underlying anatomical problems and applying the appropriate surgical and non-surgical procedures to achieve the desired result. For clinical evaluation and treatment, neck anatomy may be conceptualized into three layers wherein the superficial layer consists of the skin and subcutaneous fat, the intermediate layer of platysma muscle and interplatysmal fat, and the deep layer of subplatysmal fat, digastric muscles, submandibular glands, and skeletal support structures. The goal of this article is to review neck aesthetics, cosmetic deformities, and indications for different treatment techniques by way of a systematic layered approach.
\end{abstract}

Keywords: Neck aesthetics, cervicofacial beauty, cervicofacial aging, neck rejuvenation, neck lift, neck contouring

\section{INTRODUCTION}

An aesthetically pleasing neck is one of the defining features of a beautiful cervicofacial appearance. Without it, a beautiful face will appear unbalanced and less attractive, lacking the anatomical harmony present in youth ${ }^{[1,2]}$. Cosmetic deformities of the neck may be due to congenital or acquired etiologies, the most common of which are volume changes, platysma bands, and skin changes, all related to the aging process. For the surgeon performing a neck lift, the key to successful management is accurately diagnosing the underlying anatomical problems and applying the appropriate treatments to achieve the desired result ${ }^{[3]}$.

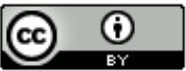

(C) The Author(s) 2021. Open Access This article is licensed under a Creative Commons Attribution 4.0 International License (https://creativecommons.org/licenses/by/4.0/), which permits unrestricted use, sharing, adaptation, distribution and reproduction in any medium or format, for any purpose, even commercially, as long as you give appropriate credit to the original author(s) and the source, provide a link to the Creative Commons license, and indicate if changes were made.

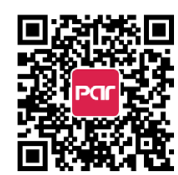


Although surgical neck rejuvenation has historically been associated with "neck lift", the traditional techniques of lifting the skin have evolved along with our understanding of the anatomical changes with aging to those of contouring the deeper neck structures to attain the most natural-looking and long-term results ${ }^{[4]}$. In turn, neck lift has become somewhat of a misnomer and the more accurate terminology may be neck recontouring. Such recontouring techniques include fat reduction or relocation, muscle plication, transection, or excision, and submandibular gland resuspension or partial resection ${ }^{[5]}$. Subsequently, the skin is redistributed on top like a drape and skin excision may be performed, if needed for real excess, in the postauricular area through a periauricular incision.

The goal of this article is to review neck aesthetics, cosmetic deformities, and indications for different treatment techniques by way of a systematic layered approach first introduced by the senior author (Nahai F) in $2005^{[6]}$.

\section{NECK AESTHETICS}

Early descriptions of the youthful female neck by Ellenbogen and Karlin ${ }^{[7]}$ were characterized by a distinct inferior mandibular border, cervicomental angle between 105 and 120 degrees, sternocleidomastoidsubmental line angle of 90 degrees, and visibility of a subhyoid depression, thyroid cartilage bulge, and anterior border of the sternocleidomastoid muscle. Later, Ramirez ${ }^{[4]}$ added to this description a welldemarcated mandibular angle influenced by lateral projection and vertical height, which Bravo ${ }^{[2]}$ explains forms a submandibular shadow important for perceived attractiveness. Ramirez noted as well that beautiful necks follow a gentler curve at the cervicomental angle and have a fatty layer that conceals the subhyoid depression and thyroid cartilage bulge, which is a more prominent feature of the male neck along with square facial shape and heavier jawline ${ }^{[8]}$. The ideal position of the chin should be tangent to Reidel's line, which connects the most projecting points of the upper and lower lips, and no more than $3 \mathrm{~mm}$ posterior to a line tangent to the most projecting points of the nose and upper lip ${ }^{[9]}$.

However, it is important to keep in mind that a "youthful neck" is not always synonymous with an aesthetic neck, as congenital anatomy or weight gain during youth can lead to deviations from the above aesthetic ideals ${ }^{[10]}$. For example, a widened cervicomental angle in a young patient may be due to microgenia, a caudally positioned hyoid bone, or excess fat ${ }^{[2,11]}$. As such, in addition to taking care of both male and female patients, the neck lift surgeon enjoys the opportunity to take care of patients of all ages.

\section{ANATOMY AND AGING}

The neck is a cylindrical structure topographically bordered inferiorly by the clavicles and superiorly by the inferior mandibular border, which is also the inferior border of the face and thus delineates the neck-face junction ${ }^{[12]}$. The anterior surface, bordered by the sternocleidomastoid muscles posteriorly, constitutes the anatomical anterior triangle and primary aesthetic unit of the neck. Within the anterior triangle, bordered by the anterior and posterior bellies of the digastric muscle and the inferior mandibular border, lies the submandibular triangle in which the submandibular gland is found.

Below the skin's surface, the anatomy of the neck and face is interconnected; the platysma muscle in the neck inserts into the perioral muscles and joins with the superficial muscular aponeurotic system (SMAS) in the face. With age, the SMAS and platysma typically descend as a single musculofascial unit and, along with the jowl fat pads, obscure the inferior mandibular border and neck-face junction. However, the morphologic process of aging is also multifactorial and affects patients heterogeneously ${ }^{[5,13]}$. For example, all other characteristics being equal, patients with shorter, heavier necks with lesser skeletal support typically develop signs of aging, in particular fullness of the neck, sooner than those with longer, thinner necks with greater skeletal support ${ }^{[4,11,14]}$. 

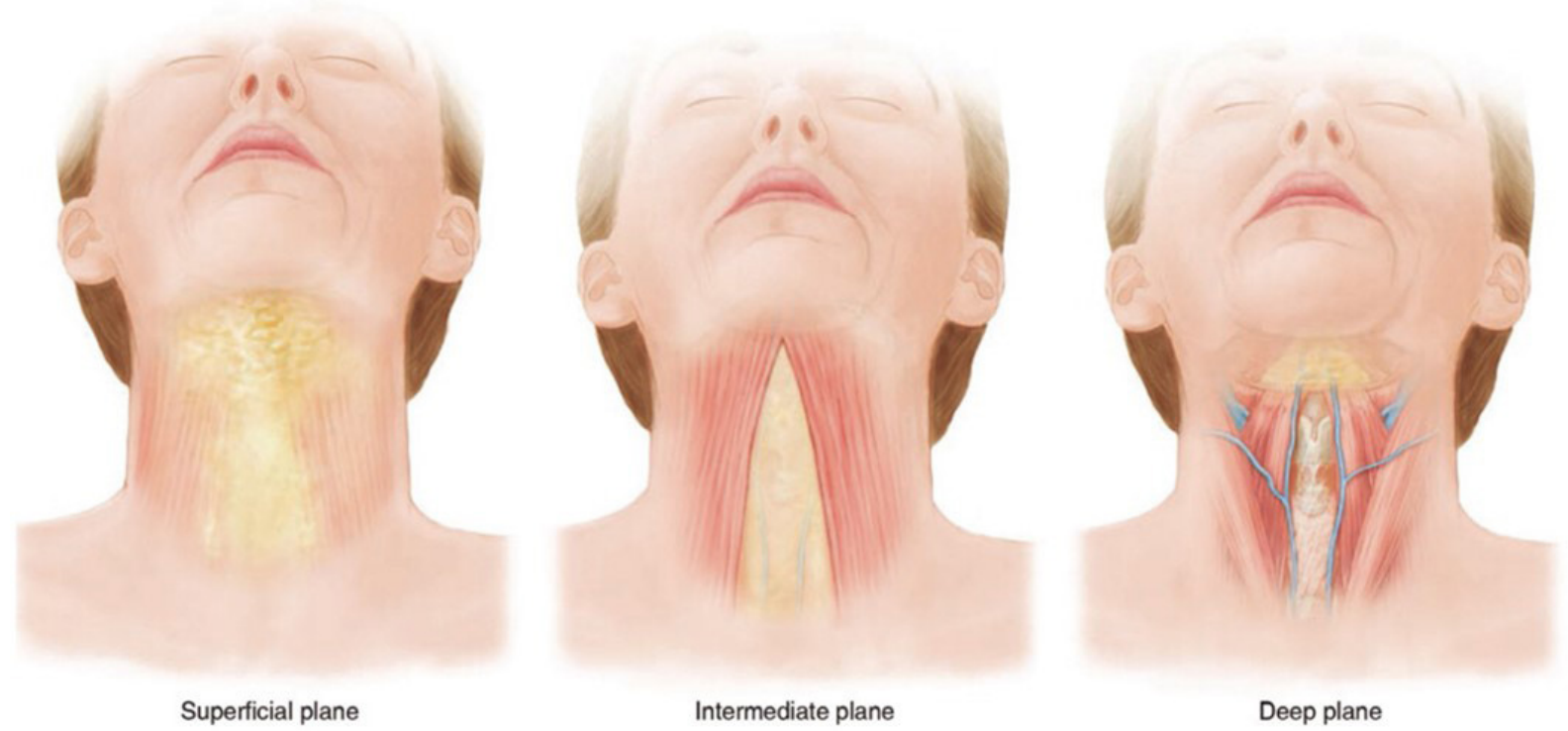

Figure 1. Three anatomical layers of the neck ${ }^{[3]}$.

Table 1. Anatomical structures in the neck by layer

\begin{tabular}{ll}
\hline Layer & Structures \\
\hline Superficial & Skin \\
& Subcutaneous fat \\
Intermediate & Platysma muscle \\
& Interplatysmal fat \\
Deep & Soft tissue \\
& Subplatysmal fat \\
& Digastric muscles \\
& Submandibular glands \\
& Skeletal support \\
& Mandible \\
& Hyoid bone \\
& Cervical vertebrae \\
\hline
\end{tabular}

As a corollary, it is imperative that the surgeon performs a complete cervicofacial analysis in order to accurately diagnose the cosmetic changes involved and the underlying anatomical changes that may be contributing to them. In general, the vast majority of anatomical changes with aging ultimately lead to widening of the cervicomental angle, either directly from skin redundancy and platysmal bands or indirectly through creating submental fullness from fat accumulation and digastric muscle and submandibular gland protrusion. For purposes of clinical evaluation and management, it is useful to conceptualize the three-dimensional anatomy of the neck as an arrangement of three layers: superficial, intermediate, and deep [Table 1, Figures 1 and 2].

\section{Superficial layer}

The superficial layer of the neck is comprised of the skin and subcutaneous fat. The skin contains collagen and elastin, which provide firmness and elasticity, respectively. In men, the skin also contains hair follicles especially in the upper half of the neck. The subcutaneous fat is located between the skin and the platysma muscle (i.e., preplatysmal fat) and occupies two compartments: suprahyoid and infrahyoid ${ }^{[15]}$. In the face, the subcutaneous fat is continuous with the jowl fat pad.

With aging, neck skin may thin from loss of collagen and elastin, a process accelerated by environmental factors such as sun exposure and smoking. Neck skin may also develop rhytids and redundancy as well 


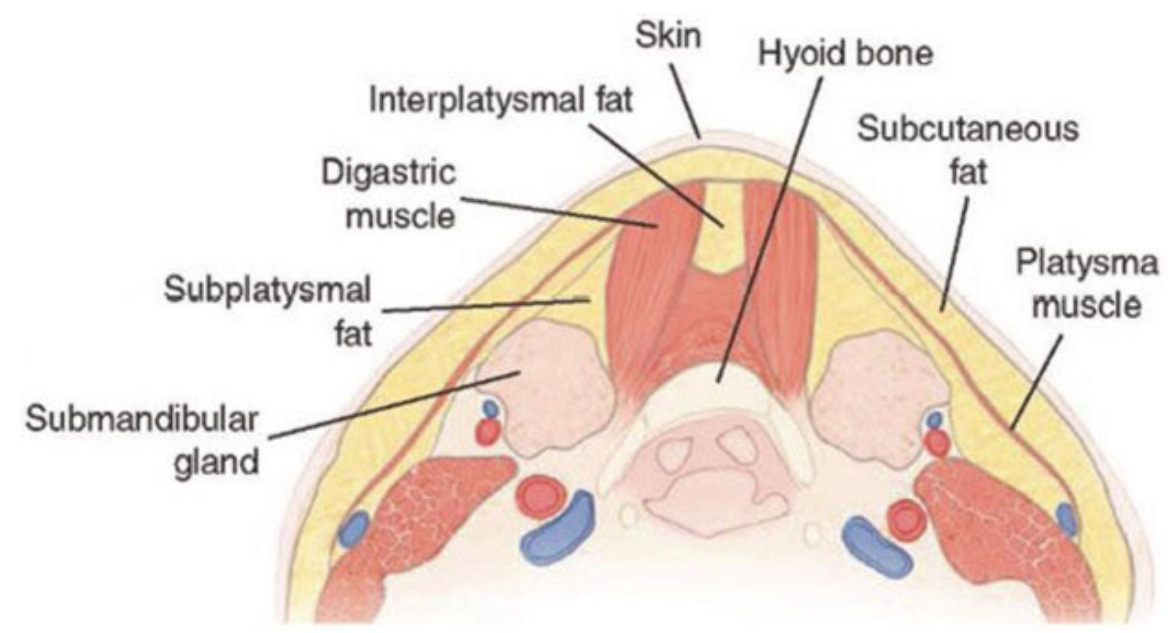

Figure 2. Cross-sectional (coronal) view of the neck ${ }^{[3]}$.

as transverse neck lines, which may become deeply etched in certain patients ${ }^{[11]}$. Subcutaneous fat may also accumulate to increase submental fullness and jowl fat pad descend to efface the inferior mandibular border.

\section{Intermediate layer}

The intermediate layer is comprised of the platysma muscle and, if present, the interplatysmal component of subplatysmal fat. The platysma is a wide, flat muscle that originates from the deltopectoral fascia on the chest, spans the neck where it is suspended to the deep cervical fascia by retaining ligaments, and inserts into the mandible, orbicularis oris muscle, and modiolus before joining with the SMAS in the face ${ }^{[16,17]}$. The muscle is innervated by the cervical branch of the facial nerve and functions at its cephalic end to pull down the oral commissures and lower lip. Interestingly, however, the platysma at its caudal end functions to pull up the lower neck skin, demonstrating that the muscle has true bidirectional movement.

In the submental region between mandibular symphysis and thyroid cartilage, the platysma muscle has variable midline decussation patterns, which have been classified by Cardoso de Castro ${ }^{[18]}$ into three types: type I (decussation for 1-2 $\mathrm{cm}$ posterior to the mandibular symphysis); type II (decussation from mandibular symphysis to thyroid cartilage); and type III (no midline decussation of platysma muscle). For patients with either type I or type III decussation patterns, a gap between medial muscle edges allows for protrusion of fat from beneath. The interplatysmal fat is therefore continuous with the deeper subplatysmal fat, which is more fibrous in consistency than subcutaneous fat ${ }^{[11]}$.

With aging, the cervical retaining ligament and the platysma muscle may develop laxity and the bands, which can be present at rest (i.e., static) or on animation (i.e., dynamic) or both ${ }^{[11]}$. Additionally, interplatysmal fat may accumulate and protrude.

\section{Deep layer}

The deep layer of the neck includes both soft tissue and skeletal support structures.

\section{Soft tissue}

The deep layer of soft tissue structures is comprised of the subplatysmal fat, digastric muscles, and submandibular glands. The subplatysmal fat is located between the platysma and submental musculature and occupies six compartments: central suprahyoid/interdigastric, central infrahyoid, lateral suprahyoid 
(bilateral), and lateral infrahyoid (bilateral) ${ }^{[15]}$. As mentioned previously, subplatysmal fat may protrude between the medial platysmal edges and has a fibrous consistency.

The paired digastric muscles originate as the posterior belly from the mastoid process of the temporal bone, transition into the intermediate tendon as it passes through a fibrous pulley attached to the hyoid bone, and then continues as the anterior belly, which inserts into the digastric fossa on inner surface of the mandible. The muscle has dual innervation with the posterior belly innervated by the digastric branch of the facial nerve and anterior belly by the mandibular branch of the trigeminal nerve and function together to elevate the hyoid bone.

The submandibular glands are located bilaterally within the submandibular triangle of the neck and are divided by the mylohyoid muscle into deep and superficial lobes, with the deep lobe being much larger in size. As a major salivary gland, the submandibular glands are the primary producers of saliva during the resting state and responsible for $20 \%-30 \%$ of total saliva produced each day ${ }^{[19]}$.

With aging, subplatysmal fat may accumulate, digastric muscles hypertrophy, and submandibular gland become ptotic or hypertrophy ${ }^{[2,1]}$.

\section{Skeletal support}

The deep layer of skeletal support structures is comprised of the mandible, hyoid bone, and cervical vertebrae. The mandible is one of the insertion sites of the platysma muscle and suspends the suprahyoid muscles of the neck (digastric, geniohyoid, and mylohyoid muscles) and, through attachments to the hyoid bone, the infrahyoid strap muscles (sternohyoid, sternothyroid/thyrohyoid, and omohyoid muscles). Ideally positioned at the level of the third vertebra, the hyoid is a horseshoe-shaped bone that coincides with vertex of the cervicomental angle ${ }^{[11]}$. It is secured in position by the peri-hyoid fascia, a condensation of deep cervical fascia of variable thickness, which may contribute to a widened cervicomental angle $e^{[19]}$. The mandible sits in the mandibular fossa of the temporal bone and articulates via temporomandibular joint. It is widest at the angle and most anteriorly projecting at the point of the chin, with the line between these points defining the jawline. Lastly, the neck is stabilized by seven cervical vertebrae with intervertebral discs between them. Together, these osseous structures serve a supportive frame for the neck.

With aging, the cervical spine shortens through compression of vertebral bodies and intervertebral discs, displacing downward the hyoid bone and deep neck soft tissue structures and increasing submental fullness through protrusion of subplatysmal fat, digastric muscles, and submandibular glands ${ }^{[14]}$. Additionally, the mandible experiences bone resorption and narrowing in both length and width, predisposing to lower facial soft tissue descent and effacement of the inferior mandibular border ${ }^{[1,20]}$.

\section{PREOPERATIVE ASSESSMENT}

\section{History}

Given the anatomical complexities in the cervicofacial region and variable changes with aging, taking a detailed patient history to clearly define the patient's aesthetic goals is critical for informing patient education and treatment choice. Such is the common case of an older patient with prominent jowls and blunting of the face-neck interface but is bothered most by the appearance of his or her neck and "only wants a neck lift". The surgeon must recognize that an isolated neck lift is unlikely to satisfactorily achieve the desired result and that an informative discussion about performing a concomitant facelift is needed to produce cervicofacial harmony. Of note, while men may be reluctant to undergo a facelift due to social stigma, Marten and Elyassnia ${ }^{[1]}$ points out that they more readily agree to undergo an "extended neck lift" procedure, which includes a periauricular incision to allow for resuspension of the lower facial soft tissues. For patients who nonetheless remain set on having an isolated neck lift after this discussion, all efforts 
should be made to establish reasonable expectations in order to ensure a successful outcome for the patient and surgeon alike.

\section{Physical exam}

In conjunction with the patient history, a careful physical exam is vital to identifying the anatomical etiologies of the cosmetic deformities observed and determining the best procedures for each patient. Beginning cephalad, the lower third of the face should be evaluated for descent of soft tissues presenting as effacement of the inferior mandibular border and prominent jowls. As described previously, the presence of these exam findings indicates that a neck lift procedure alone would likely be insufficient to produce a harmonious aesthetic result and a concomitant facelift is indicated.

Moving to the neck assessment, a layered approach is applied starting superficially and working deep. In general, cosmetic neck deformities can be divided into four main categories: skin redundancy, submental fullness, platysmal bands, and prominent submandibular glands. Skin redundancy may be classified into two clinically relevant subtypes: "apparent" or "real" skin excess ${ }^{[3]}$. Conceptualizing the aesthetic cervicomental angle as two short sides of a triangle and the "turkey neck" as one long side (i.e., the hypotenuse), apparent skin excess exists when, after the deeper layers of the neck are appropriately contoured, redraping skin of the "long side" onto the two shorter sides does not result in dermatochalasis ${ }^{[1]}$. If dermatochalasis persists, however, then the skin redundancy is real and a periauricular incision and excision in the postauricular area will be needed to address it. Skin quality also plays an important role in differentiating, as patients with good skin elasticity are more likely than those with sun-damaged or crepey skin to fall into the apparent excess group.

Next, the platysma muscle should be examined not only for platysmal bands, but their location (medial $v s$. lateral) and relationship with animation (static vs. dynamic) as well. Whereas static bands are present at rest, dynamic bands must be determined by palpating the muscle accordingly and asking the patient to grimace. If medial bands are present, the distance between their medial edges should be noted for surgical planning.

Submental fullness should then be investigated as it may result from multiple anatomical etiologies including excess subcutaneous and subplatysmal fat and hypertrophy of the digastric muscles and submandibular glands. Flexing the neck is a helpful provocative maneuver both to evaluate the deep structures of the neck and establish a baseline exam for dynamic changes that occur with movement ${ }^{[2,5]}$. To differentiate between subcutaneous and subplatysmal fat, a pinch test can be performed wherein the surgeon pinches the submental area with the neck muscles at rest and observes the degree to which this soft tissue bulk diminishes upon platysma muscle contraction, with significant diminishment indicating the presence of a deeper source. Although digastric muscle and submandibular gland enlargement may be visualized or palpated on exam, often these deeper structures are camouflaged by the overlying soft tissue layers and can only be fully appreciated intraoperatively once the platysma muscle has been elevated.

Lastly, the skeletal support structures should be evaluated for the presence of microgenia or a caudally positioned hyoid bone, which are more common findings in younger patients with cosmetic neck deformities.

\section{INTERVENTION BY PLANE}

Treatments to improve neck aesthetics include both surgical and non-surgical options, which are often combined in comprehensive neck rejuvenation to attain the most natural-looking and long-term results. Overall, we recommend using a systematic layered approach with the goal of modifying the precise 
Table 2. Surgical treatment options based on patient anatomical characteristics

\begin{tabular}{|c|c|c|c|c|c|c|c|c|c|}
\hline \multirow[b]{2}{*}{ Procedure } & \multirow{2}{*}{ Incision(s) } & \multicolumn{3}{|c|}{ Skin excess } & \multicolumn{2}{|c|}{ Skin elasticity } & \multicolumn{2}{|c|}{ Fat excess } & \multirow{2}{*}{$\begin{array}{c}\text { Mandibular } \\
\text { border } \\
\text { effacement/ } \\
\text { jowls }\end{array}$} \\
\hline & & None & Apparent & Real & Good & Poor & Subcutaneous & Subplatysmal & \\
\hline $\begin{array}{l}\text { Isolated } \\
\text { liposuction }\end{array}$ & $\begin{array}{l}\text { Access (stab) } \\
\text { incisions }\end{array}$ & $\sqrt{ }$ & & & $\sqrt{ }$ & & $\sqrt{ }$ & & \\
\hline $\begin{array}{l}\text { Submental } \\
\text { neck lift }\end{array}$ & Submental & $\sqrt{ }$ & $\sqrt{ }$ & & $\sqrt{ }$ & & $\sqrt{ }$ & $\sqrt{ }$ & \\
\hline $\begin{array}{l}\text { Short-scar } \\
\text { neck lift }\end{array}$ & $\begin{array}{l}\text { Retroauricular } \pm \\
\text { submental }\end{array}$ & & & $\sqrt{ }$ & & $\sqrt{ }$ & $\sqrt{ }$ & $\sqrt{ }$ & \\
\hline $\begin{array}{l}\text { Full-scar face- } \\
\text { and neck lift }\end{array}$ & $\begin{array}{l}\text { Periauricular } \pm \\
\text { submental }\end{array}$ & & & $\sqrt{ }$ & & $\sqrt{ }$ & $\sqrt{ }$ & $\sqrt{ }$ & $\sqrt{ }$ \\
\hline
\end{tabular}

Table 3. Our indications for opening the neck through a submental incision

\begin{tabular}{ll}
\hline Indication & \multicolumn{1}{c}{ Intervention } \\
\hline Excess, inelastic central neck skin & Skin undermining and redraping \\
Deeply etched transverse neck lines & Skin undermining and redraping \\
Excess inter- or subplatysmal fat & Resection \\
Visible medial platysmal bands (<3 cm apart) & Medial plication with myotomy or myectomy \\
Static bands & Partial myotomy \\
Dynamic bands & Partial myotomy or myectomy \\
Recurrent or recalcitrant bands & Total myotomy or myectomy \\
Prominent digastric muscles & Plication or resection \\
Prominent submandibular glands & Resection \\
\hline
\end{tabular}

anatomical structures causing the cosmetic deformities and avoiding contour abnormalities, which may occur from overcorrection of the superficial layer and undercorrection of the deep layer ${ }^{[21]}$.

Although the approach to neck rejuvenation should always be customized to the patient's individual anatomy and aesthetic goals, there are several common surgical indications that may be used as general guidelines [Tables 2 and 3$]^{[3,22,23]}$.

\section{Superficial plane}

Cosmetic deformities of the superficial plane consist mainly of skin surface irregularities, such as solar lentigines and rhytids, skin redundancy, and excess fat in the subcutaneous layer. Given their accessible location, the skin and subcutaneous tissues are frequently targeted by non-surgical modalities. To improve skin surface irregularities, the mainstay of treatment is resurfacing with chemical peel and laser treatments, and microneedling techniques have been shown to improve skin quality as well ${ }^{[24]}$. Skin resurfacing is also routinely combined with surgical treatments.

For younger patients with submental fullness due primarily to excess subcutaneous fat, radiofrequency, ultrasound, and cryolipolysis devices and deoxycholic acid injections may be used to reduce the fat and tighten the overlying skin ${ }^{[25]}$. Although non-surgical, these modalities are still associated with risks, however, such as skin burns and nerve injuries with energy-based devices, and often require multiple treatments to achieve modest effects ${ }^{[26,27]}$. For example, there have been reports of radiofrequency devices causing submental contour irregularities and scarring that may make dissection during future neck lift surgery more challenging and the complication risk greater ${ }^{[2,21]}$. Consequently, the senior author (Nahai F) prefers using liposuction, which is thought to be safer and more effective when no less than 3-5 $\mathrm{mm}$ of subcutaneous fat is left on the skin flap, and anatomically limiting the lipectomy to the mandibular border superiorly, anterior border of the sternocleidomastoid muscle laterally, and hyoid bone inferiorly [Figure 3]. For isolated liposuction, patient selection is key as its use in the wrong patient could be counterproductive, leading to worsened skin redundancy and potential exposure of platysmal bands, digastric muscles, and submandibular glands. 

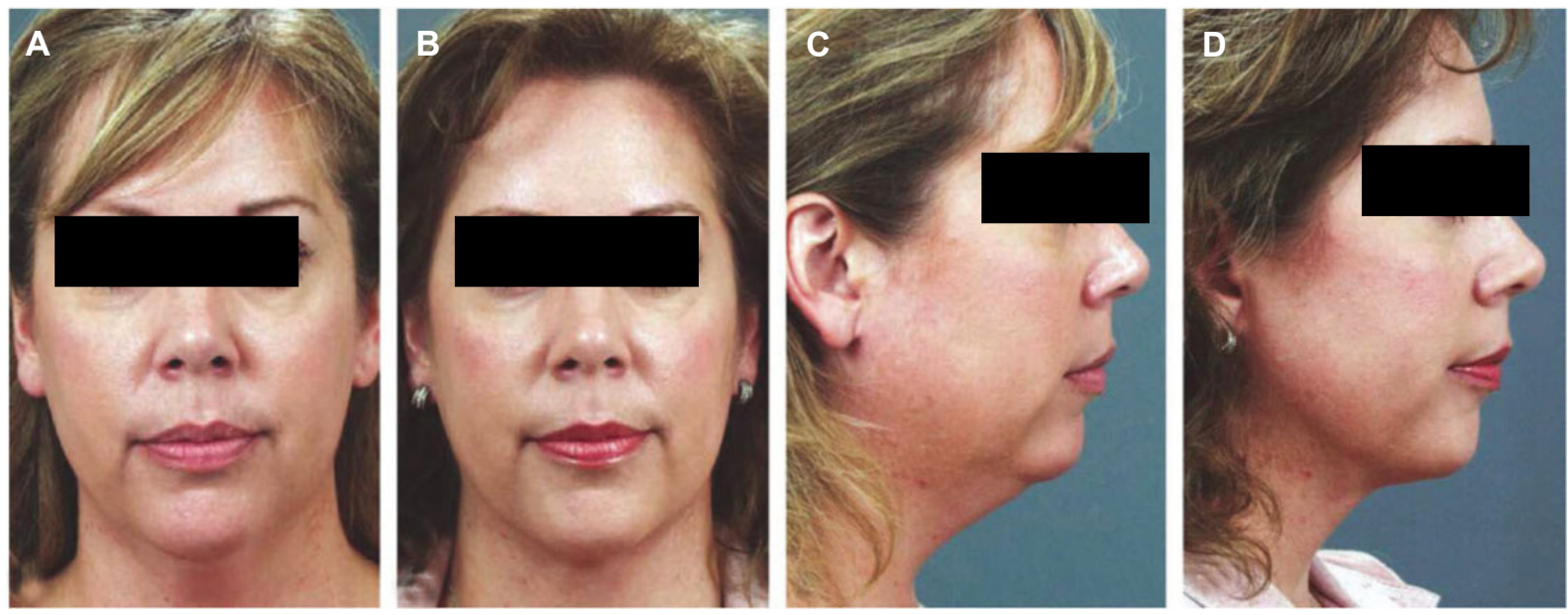

Figure 3. (A, C) Preoperation and (B, D) 4-year postoperation photos following liposuction of the neck without skin excision ${ }^{[3]}$.
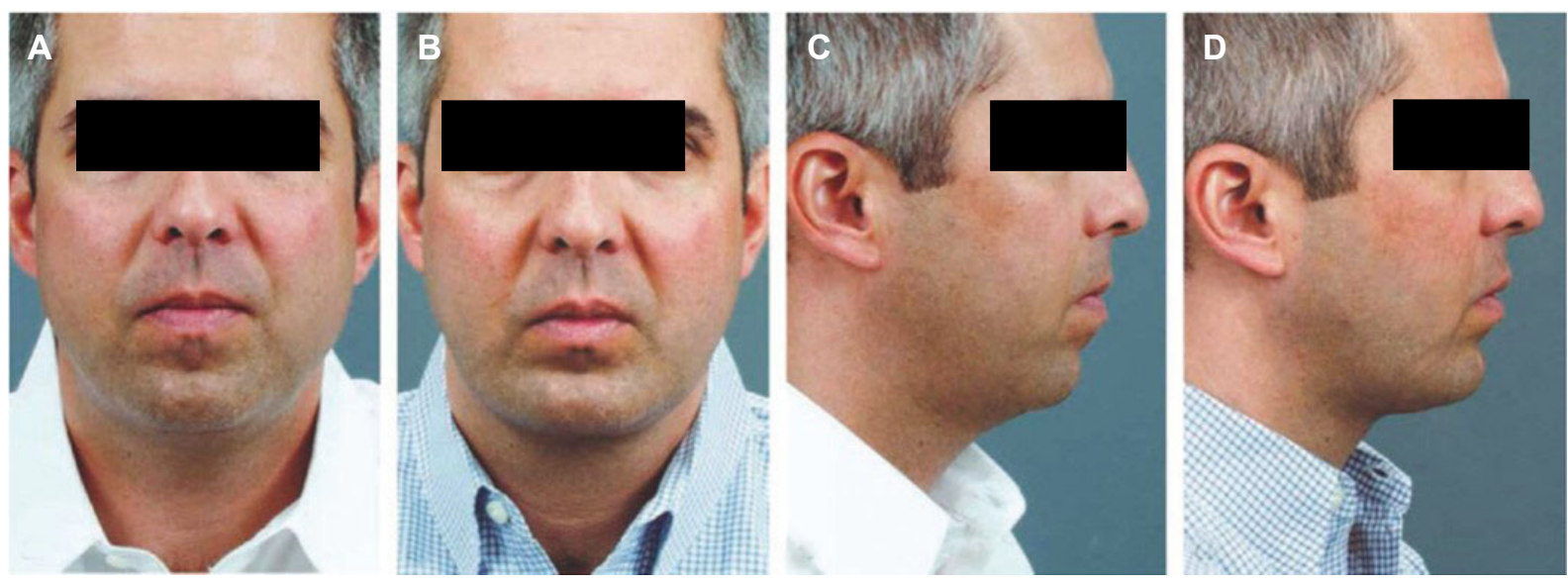

Figure 4. $(A, C)$ Preoperation and $(B, D)$ postoperation photos following submental neck lift, including subplatysmal fat and digastric excision, and chin augmentation ${ }^{[3]}$.

For patients with real skin excess, however, surgical excision in the postauricular area through a retroauricular incision is needed for optimal management. In such cases, if the deeper neck structures will be contoured as part of the neck lift, then lipectomy of the subcutaneous layer is done afterward in order to minimize the risk of causing a submental depression and skeletonized appearance ${ }^{[3]}$.

\section{Intermediate plane}

Cosmetic deformities of the intermediate plane involve platysmal bands and any subplatysmal fat herniating between medial edges of the platysma muscle as interplatysmal fat. In contrast to subcutaneous fat, interplatysmal fat has a more fibrous consistency less amenable to liposuction and should instead be resected via surgical lipectomy. Although dynamic platysmal bands can be treated non-surgically with botulinum toxin, albeit with repeated injections every 3-4 months, a submental neck lift through a submental incision is needed for long-term treatment and to correct platysma muscle laxity manifesting as bands [Figure 4$]^{[10,28,29]}$.

Numerous surgical manipulations of the platysma muscle have been described with the common goal to eliminate platysmal bands, reduce submental fullness, and improve the cervicomental angle. Medial platysmal bands are usually managed with anterior platysmaplasty, various medial plication techniques of 
which have been built upon Feldman's original description of the corset platysmaplasty ${ }^{[30-32]}$. For patients in whom the distance between platysma muscle edges is greater than $3 \mathrm{~cm}$, medial plication may not be possible and platysmal suspension via plication through a lateral approach is indicated ${ }^{[27]}$.

During management of platysmal bands, the platysma muscle may be transected as a myotomy, wedge myectomy, or not transected at all depending on the degree and visibility of the bands at rest and on animation ${ }^{[31,32]}$. Static bands may be treated with horizontal myotomy that is placed low at the level of the cricoid to avoid affecting the lip depressor function and masculinizing the neck unmasking the thyroid cartilage prominence, followed by midline plication ${ }^{[5,8]}$. Dynamic bands, on the other hand, have been suggested to be more effectively treated with wedge myectomy of medial platysmal muscle ${ }^{[31]}$. Of note, successful long-term treatment of platysmal bands remains a challenge and clinically enigmatic, as recurrence has been shown to occur in over one-third of cases despite complete transection of the muscle ${ }^{[33]}$. As a result, in addition to muscle transection, it is recommended that the fascia on its posterior surface be released as well ${ }^{[33]}$. In all cases, any excess interplatysmal fat should be excised prior to midline plication.

Although it is possible to manipulate lateral platysmal bands through a submental incision, they are more easily suspended using a lateral approach through a retroauricular incision. First described by Cruz et al. ${ }^{[34]}$, the lateral platysmal window is one such technique employed to anchor the platysma muscle to the mastoid fascia. This lateral access is already available if either a short-scar neck lift or full-scar face and neck lift is being performed to address real skin excess or lower facial soft tissue descent, respectively. In such cases, the senior author (Nahai F) invariably plicates the platysma laterally in combination with SMAS management.

\section{Deep plane}

Cosmetic deformities of the deep plane include excess subplatysmal fat, digastric muscle hypertrophy, and submandibular gland ptosis or hypertrophy. As previously mentioned, these neck structures may be covered by thick and redundant soft tissues and are therefore difficult to assess on physical exam. Thus, when a source of submental fullness is determined on pinch test to be subplatysmal, it is recommended that the platysma muscle be opened through an anterior approach and underlying structures assessed under direct visualization.

Excess subplatysmal fat should be resected only enough to be flush with the superficial border of the digastric muscles, as more aggressive resection in the submental area can lead to a submental depression and dreaded cobra deformity, which is very difficult to correct ${ }^{[1,16,32]}$. If the anterior bellies of the digastric muscles appeared hypertrophied, they may be reduced by tangential excision as originally described by Connell and Shamoun ${ }^{[35]}$. Alternatively, a midline plication of the anterior bellies may be performed, socalled digastric corset as popularized by Labbé et al. ${ }^{[36]}$, but this technique works best when the muscles are only moderately enlarged.

Management of the submandibular glands should begin by determining whether the observed prominence is due to gland ptosis or hypertrophy or both, as attempts at tightening the platysmal muscle over prominent glands may counterproductively make those glands more conspicuous ${ }^{[1,31]}$. For ptotic submandibular glands, a simple resuspension of the gland capsule may be performed. However, for the more frequent scenario of gland hypertrophy, a partial submandibular gland resection of the superficial lobe remains the most effective and definitive treatment [Figure 5 $]^{[1,2,32,37,38]}$. Although this procedure continues to be controversial among surgeons due to the small, but dangerous risk of airway compromise from postoperative arterial bleeding beneath the deep cervical fascia, it may still be performed safely and reliably by experienced surgeons ${ }^{[38,39]}$. In a retrospective study by Auersvald of 504 patients who 

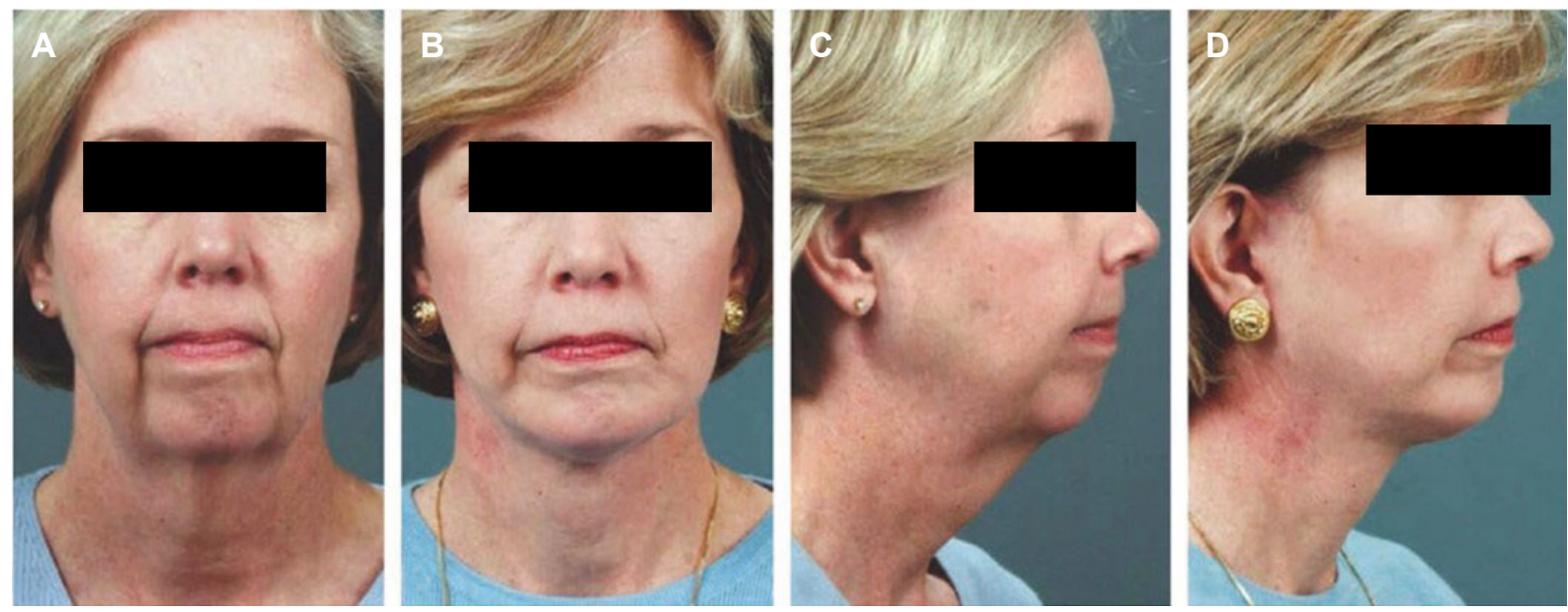

Figure 5. $(A, C)$ Preoperation and (B, D) postoperation photos following facelift and submental neck lift, including deep plane procedures, subplatysmal fat and digastric excision with no subcutaneous fat excision ${ }^{[3]}$.

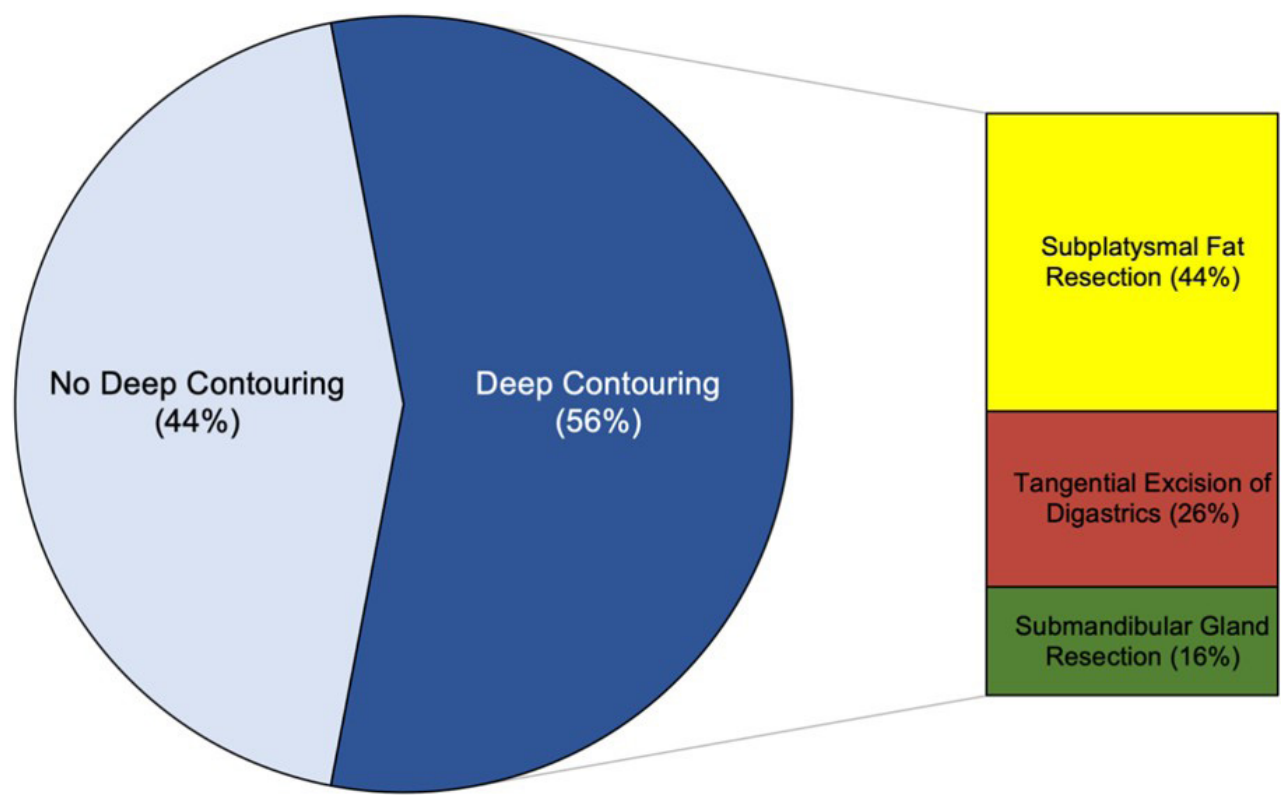

Figure 6. Breakdown of deep neck contouring procedures performed by the senior author (Nahai F) among 56 consecutive subplatysmal neck lifts.

underwent subplatysmal neck lift, 307 (61\%) had submandibular gland resection. While 5.8\% of this cohort experienced transient lower lip depressor weakness and $0.4 \%$ submandibular gland sialoma, there were no instances of subplatysmal hematoma ${ }^{[40]}$. In an unpublished series of 100 consecutive neck lifts performed by the senior author (Nahai F) between 2003 and 2005, subplatysmal procedures were undertaken in 56\% of cases, of which $16 \%$ involved partial submandibular gland resection, and there were no postoperative instances of subplatysmal hematoma, permanent marginal mandibular nerve injury, dry mouth, or radical appearance of the neck (study findings presented at the annual meeting of the American Society for Aesthetic Plastic Surgery in 2005 by Dr. Farzad Nahai) [Figure 6]. Nevertheless, all known risks of gland resection including bleeding, sialocele, salivary fistula, and dry mouth symptoms must be discussed with the patient preoperatively and she or he given the opportunity to forgo this portion of the procedure. For patients who opt out, an alternative treatment is shrinking the gland with a series of botulinum toxin 
injections, keeping in mind that the effect cannot be assessed immediately and final gland morphology can be unpredictable $e^{[22,41]}$.

Lastly, there are several important management considerations for the skeletal support structures that form the frame of the cervicomental angle. For patients with inadequate chin projection due to microgenia, a chin implant can be placed to improve mandibular border aesthetics and the cervicomental angle ${ }^{[1]}$. In the absence of microgenia, however, placing a chin implant may actually create an unnatural appearance and should be avoided ${ }^{[1]}$. A deficient mandibular border can be augmented with fillers, such hyaluronic acid or calcium hydroxylapatite, autologous fat grafting or tissue flaps, and prosthetic implants ${ }^{[20]}$. For patients with a caudally positioned hyoid bone, the peri-hyoid fascial attachments to the bone may be released in order to allow the latter to migrate superoposteriorly to both deepen and sharpen the cervicomental angle $e^{[19,32,37]}$. Together with the aforementioned soft tissue procedures, optimization of the skeletal framework will further enhance the final aesthetic result and neck lift longevity, and positively contribute to the shared goal of the patient and surgeon.

\section{CONCLUSION}

Overall, there are many non-surgical and surgical options available for improving the appearance of cosmetic neck deformities, ranging from skin resurfacing to deep neck contouring. While most patients presenting for neck lift desire neck rejuvenation from the sequelae of aging, others may have a congenital etiology that has bothered them since childhood. In all cases, the key to successful management depends on accurately diagnosing the underlying anatomical problems and applying the appropriate treatments to achieve the desired result. Given the anatomical complexity and variability of cosmetic neck deformities, we recommend following a systematic layered approach for their clinical evaluation and treatment.

\section{DECLARATIONS}

\section{Authors' contributions}

Made substantial contributions to the conception and writing of this manuscript: Weinstein AL, Nahai F

\section{Financial support and sponsorship}

None.

\section{\$ YDITELLON $\backslash R I$ IGDUDIDQGIP DMADQ}

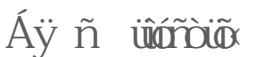

प

\section{Conflicts of interest}

Both authors declared that there are no conflicts of interest.

口

Ethical approval and consent to participate

Not applicable.

प

\section{Consent for publication}

Not applicable.

\section{प}

\section{Copyright}

(9) The Author(s) 2021

\section{REFERENCES}

1. Marten T, Elyassnia D. Neck lift: defining anatomic problems and choosing appropriate treatment strategies. Clin Plast Surg 2018;45:45584.

2. Bravo FG. Reduction neck lift: the importance of the deep structures of the neck to the successful neck lift. Clin Plast Surg 2018;45:485506.

3. Nahai F. Neck lift. In: Nahai F, editor. The art of aesthetic surgery: principles $\&$ techniques. $3^{\text {rd }}$ ed. Thieme Medical Publishers, Inc.; 2020. p. $749-70$. 
4. Ramírez OM. Advanced considerations determining procedure selection in cervicoplasty. Part one: anatomy and aesthetics. Clin Plast Surg 2008;35:679-90, viii.

5. Mejia JD, Nahai FR, Nahai F, Momoh AO. Isolated management of the aging neck. Semin Plast Surg 2009;23:264-73.

6. Nahai F. Clinical decision-making in face lift and neck lift. In: Nahai F, editor. The art of aesthetic surgery: principles \& techniques. 1st ed. Quality Medical Publishing, Inc.; 2005. p. 1347-76.

7. Ellenbogen R, Karlin JV. Visual criteria for success in restoring the youthful neck. Plast Reconstr Surg 1980;66:826-37.

8. Marten T, Elyassnia D. Short scar neck lift: neck lift using a submental incision only. Clin Plast Surg 2018;45:585-600.

9. Metzinger S, Lettieri S, Guerra A. Rejuvenation of the cheeks, chin, lips, and ears. In: Nahai F, editor. The art of aesthetic surgery: principles \& techniques. Thieme Medical Publishers, Inc.; 2020. p. 882-94.

10. Huettner F, Vasconez LO, de la Torre JI. Neck rejuvenation-anatomy and clinical correlation. Facial Plast Surg 2012;28:40-51.

11. Shadfar S, Perkins SW. Anatomy and physiology of the aging neck. Facial Plast Surg Clin North Am 2014;22:161-70.

12. Kohan EJ, Wirth GA. Anatomy of the neck. Clin Plast Surg 2014;41:1-6.

13. de Castro CC, Aboudib JH Jr, Roxo ACW. Updating the concepts on neck lift and lower third of the face. Plast Reconstr Surg 2012;130:199-205.

14. Ramirez OM. Multidimensional evaluation and surgical approaches to neck rejuvenation. Clin Plast Surg 2014;41:99-107.

15. Larson JD, Tierney WS, Ozturk CN, Zins JE. Defining the fat compartments in the neck: a cadaver study. Aesthet Surg J 2014;34:499506.

16. Charafeddine AH, Couto RA, Zins JE. Neck rejuvenation: anatomy and technique. Clin Plast Surg 2019;46:573-86.

17. Labbé D, Rocha CSM, de Souza Rocha F. Cervico-mental angle suspensory ligament: the keystone to understand the cervico-mental angle and the ageing process of the neck. Aesthetic Plast Surg 2017;41:832-6.

18. Cardoso de Castro C. The value of anatomical study of the platysma muscle in cervical lifting. Aesthetic Plast Surg 1984;8:7-11.

19. O'Daniel TG. Understanding deep neck anatomy and its clinical relevance. Clin Plast Surg 2018;45:447-54.

20. Jacono AA, Bryant LM, Ahmedli NN. A novel extended deep Plane facelift technique for jawline rejuvenation and volumization. Aesthet Surg $J$ 2019;39:1265-81.

21. Gordon NA, Paskhover B, Tower JI, O’Daniel TG. Neck deformities in plastic surgery. Facial Plast Surg Clin North Am 2019;27:529-55.

22. Matarasso A. Managing the components of the aging neck: from liposuction to submentalplasty, to neck lift. Clin Plast Surg 2014;41:8598.

23. Nahai F. Clinical decision making in facelift and neck lift. In: Nahai F, editor. The art of aesthetic surgery: principles \& techniques. 3rd ed. Thieme Medical Publishers, Inc.; 2020. p. 529-50.

24. Kislevitz M, Nahai F, Kenkel JM. Clinical decision making for nonsurgical cosmetic treatments. In: Nahai F, editor. The art of aesthetic surgery: principles \& techniques. 3rd ed. Thieme Medical Publishers, Inc.; 2020. p. 103-10.

25. Dibernardo BE. The aging neck: a diagnostic approach to surgical and nonsurgical options. $J$ Cosmet Laser Ther 2013;15:56-64.

26. Rotunda AM, Weiss SR, Rivkin LS. Randomized double-blind clinical trial of subcutaneously injected deoxycholate versus a phosphatidylcholine-deoxycholate combination for the reduction of submental fat. Dermatol Surg 2009;35:792-803.

27. Gassman AA, Pezeshk R, Scheuer JF 3rd, Sieber DA, Campbell CF, Rohrich RJ. Anatomical and clinical implications of the deep and superficial fat compartments of the neck. Plast Reconstr Surg 2017;140:405e-14e.

28. Jabbour SF, Kechichian EG, Awaida CJ, Tomb RR, Nasr MW. Botulinum toxin for neck rejuvenation: assessing efficacy and redefining patient selection. Plast Reconstr Surg 2017;140:9e-17e.

29. Kane MA. Nonsurgical treatment of platysmal bands with injection of botulinum toxin A. Plast Reconstr Surg 1999;103:656-63; discussion 664-5.

30. Feldman JJ. Corset platysmaplasty. Plast Reconstr Surg 1990;85:333-43.

31. Marten T, Elyassnia D. Management of the platysma in neck lift. Clin Plast Surg 2018;45:555-70.

32. Feldman JJ. Neck lift my way: an update. Plast Reconstr Surg 2014;134:1173-83.

33. Pelle-Ceravolo M, Angelini M, Silvi E. Complete platysma yransection in neck rejuvenation: a critical appraisal. Plast Reconstr Surg 2016;138:781-91.

34. Cruz RS, O’Reilly EB, Rohrich RJ. The platysma window: an anatomically safe, efficient, and easily reproducible approach to neck contour in the face lift. Plast Reconstr Surg 2012;129:1169-72.

35. Connell BF, Shamoun JM. The significance of digastric muscle contouring for rejuvenation of the submental area of the face. Plast Reconstr Surg 1997;99:1586-90.

36. Labbé D, Giot JP, Kaluzinski E. Submental area rejuvenation by digastric corset: anatomical study and clinical application in 20 cases. Aesthetic Plast Surg 2013;37:222-31.

37. Auersvald A, Auersvald LA. Management of the submandibular gland in neck lifts: indications, techniques, pearls, and pitfalls. Clin Plast Surg 2018;45:507-25.

38. Mendelson BC, Tutino R. Submandibular gland reduction in aesthetic surgery of the neck: review of 112 consecutive cases. Plast Reconstr Surg 2015;136:463-71.

39. Baker D. Face lift with submandibular gland and digastric muscle resection: Radical neck rhytidectomy. Aesthetic Surgery Journal 2006;26:85-92.

40. Auersvald A, Auersvald LA, Oscar Uebel C. Subplatysmal necklift: a retrospective analysis of 504 patients. Aesthet Surg J 2017;37:1-11.

41. Trevidic P. Neck contouring. Clin Plast Surg 2014;41:81-3. 